\title{
In vitro effects of artesunate on the survival of worm pairs and egg production of Schistosoma mansoni
}

\author{
Y. Mitsui*, M. Miura and Y. Aoki \\ Department of Parasitology, Institute of Tropical Medicine, Nagasaki \\ University, Sakamoto, Nagasaki, 852-8523 Japan
}

\begin{abstract}
The effect of artesunate (ART) on the survival time of adult worm pairs of Schistosoma mansoni and on their egg output during in vitro culture was assessed. ART significantly decreased the survival time of both paired male and female worms at concentrations of 5, 10, 20 and $40 \mathrm{mgl}^{-1}$ during in vitro cultivation. An inhibitory effect of ART on the daily egg output of paired female worms during in vitro cultivation was also observed.
\end{abstract}

\section{Introduction}

Schistosomiasis is one of the world's major public health problems, with an estimated 200 million people infected, mostly in Africa (Chitsulo et al., 2000). As praziquantel (PZQ) is safe and has a high efficacy against schistosomes (Magnussen, 2003), mass chemotherapy with PZQ constitutes the current major strategy for control (Montresor et al., 2002). However, this extensive reliance on just one drug is of concern, due to the possible emergence of drug-resistant parasites (Doenhoff et al., 2002). Indeed, there is already clinical evidence for the presence of PZQ-resistant schistosomes in Senegal and Egypt (Guisse et al., 1997; Ismail et al., 1999). In view of the possible emergence of resistance to PZQ, there is a need for the development of novel antischistosomal drugs.

Artemisinin (ARS) derivatives such as artemether (ARM) and artesunate (ART) are not only well known as antimalarial drugs, but also possess prophylactic activity against schistosomiasis (Utzinger et al., 2000; Xiao et al., 2000a). Furthermore, the effect on schistosomulae has been confirmed by studies in animal models (Xiao \& Catto, 1989; Xiao et al., 1995; Utzinger et al., 2002). However, these studies show that the efficacy of ARS derivatives may be low when used in monotherapy. For example, a study using an animal model showed that ARM alone reduced the number of worms in infected mice by $39 \%$, when high concentrations of the drug (1200 $\mathrm{mg} \mathrm{kg}^{-1}$ ) were administered (Xiao \& Catto, 1989). Due to the low therapeutic efficacy of ARS derivatives in

*Fax: + 81958197824

E-mail: ymitsui@nagasaki-u.ac.jp monotherapy (Borrmann et al., 2001), combination of ART with PZQ is required for a high curative effect of schistosomiasis infections (Utzinger et al., 2001).

Understanding the effects of ARS derivatives on the survival time and egg productivity of adult worm pairs of Schistosoma mansoni is important for developing an efficacious treatment regimen for schistosomiasis. Previous in vitro studies have, however, concentrated on the efficacy of these drugs against adult worms. For example, an anthelminthic effect of ARM on adult worms of $S$. mansoni was observed at drug concentrations as low as $100 \mathrm{mg} \mathrm{l}^{-1}$ but not at a concentration of $25 \mathrm{mg} \mathrm{l}^{-1}$ (Xiao \& Catto, 1989). More recently, Xiao et al. (2001) indicated that ARM exerts an anthelminthic effect through synergy with haemin at a drug concentration of $20 \mathrm{mg}^{-1}$. Furthermore, Jiraungkoorskul et al. (2006) reported that most adult worms of Schistosoma mekongi died $24 \mathrm{~h}$ after in vitro exposure of worms to $40 \mathrm{mg} \mathrm{l}^{-1}$ ART. To date, there are no reports detailing the effect of low concentrations of ART as a monotherapy on S. mansoni adult worms in vitro.

The objective of the present study is to investigate the effect of ART monotherapy on the survival time of adult worm pairs of $S$. mansoni and their daily egg output during in vitro incubation at drug concentrations of 5,10 , 20 and $40 \mathrm{mgl}^{-1}$.

\section{Materials and methods \\ Chemicals and media}

ART was purchased from LKT laboratories, Inc. (St. Paul, Minnesota, USA) and diluted in ethanol to a concentration of $20 \mathrm{mg} \mathrm{l}^{-1}$ as a stock solution. This stock ART solution was 
then added to medium with an ethanol concentration of $0.2 \%$ (v/v). NCTC 135 medium (Sigma, St Louis, Missouri, USA) containing 1\% solution of antibiotics (penicillin 5000 units and streptomycin $5000 \mathrm{mgl}^{-1}$; Gibco, Langley, Oklahoma, USA) was used for in vitro culture.

\section{Parasite strain}

A Puerto Rican strain of S. mansoni (NIH-Sm-PR-1 strain) was routinely maintained by passage through GN hamsters and Biomphalaria glabrata (Newton's NIH Puerto Rican/Brazilian M-line) snails. At 8 weeks post-infection, adult worms were obtained by the perfusion technique, as described previously (Smithers \& Terry, 1965), and washed with NCTC 135 medium twice.

\section{Incubation}

In Experiment 1, 48 male/female adult worm pairs of $S$. mansoni were randomly assigned into three equally sized groups: control, 20 and $40 \mathrm{mg}^{-1}$ ART groups. Each worm pair was preincubated for 1 day in a single well of a 24-well multi-well plate (Sumitomo bakelite Co. Ltd, Japan) with $0.5 \mathrm{ml}$ of NCTC 135 medium alone in a $5 \%$ $\mathrm{CO}_{2}$ incubator at $37^{\circ} \mathrm{C}$.

Each worm pair was subsequently transferred into a well containing $0.5 \mathrm{ml}$ of NCTC 135 medium containing $0.2 \%$ ethanol alone for controls, or $0.2 \%$ ethanol supplemented with 20 or $40 \mathrm{mg} \mathrm{l}^{-1}$ ART. Media were exchanged every day for 8 days. The number of eggs produced daily was counted and worm viability was observed visually under a Nikon SMZ 800 stereoscopic microscope. Assessment of 'dead or alive' status of adult worms was determined by the movement response of the worm upon stimulation with a needle. Worms were classified as 'dead' when they failed to respond to needle stimulation.

For Experiment 2, the procedure was identical to that described for the first experiment, except for the concentration of ART and the incubation period. Twenty-seven adult worm pairs were equally distributed between three groups and were pre-incubated in NCTC 135 medium for $24 \mathrm{~h}$. Subsequently, each group was incubated in medium containing $0.2 \%$ ethanol alone or $0.2 \%$ ethanol supplemented with 5 and $10 \mathrm{mg}^{-1}$ ART for 21 days, and parasite response recorded as above.

\section{Statistical analysis}

Data were analysed using Epi-Info software (Centers for Disease Control and Prevention, Atlanta, Georgia, USA). A log-rank test was used to compare the survival times of worms. Daily egg output per female adult worm was expressed as the arithmetic mean ( \pm standard error). The Mann-Whitney test was used for comparing daily egg outputs between ART-treated groups and controls.

\section{Results}

The effect of artesunate on the survival time of Schistosoma mansoni adult worm pairs

The survival of adult worm pairs of $S$. mansoni was recorded during in vitro incubation with various concentrations of ART. The survival times of adult worm pairs incubated with 20 and $40 \mathrm{mgl}^{-1}$ ART (Experiment 1) and 5 and $10 \mathrm{mgl}^{-1}$ ART (Experiment 2) are shown in table 1. In Experiment 1, 2 out of 16 pairs in the control group separated within 1 or 2 days after incubation and all other worms remained paired throughout the experimental duration (8 days). No worms in the control group died during the experimental duration. Worm pairs incubated with 20 and $40 \mathrm{mgl}^{-1}$ ART separated or died with a median duration of 3 and 2 days of exposure to ART, respectively. ART significantly reduced the survival times of male (median: 3 days, $P<0.01$ ) and female (median: 3 days, $P<0.01$ ) worms at a concentration of $40 \mathrm{mgl}^{-1}$ when compared with the control group. ART also significantly reduced the survival times of male (median: 6 days, $P<0.01$ ) and female (median: 4 days, <0.01) worms at a concentration of $20 \mathrm{mg} \mathrm{l}^{-1}$.

In Experiment 2, adult worm pairs of $S$. mansoni were cultivated with 5 and $10 \mathrm{mgl}^{-1}$ ART for 21 days. In the control group, 1 pair among 9 worm pairs separated 4 days after exposure to control medium, and all other worms remained paired throughout the observation duration. All worms survived for the 21 days of the experiment. All worm pairs incubated with 5 and $10 \mathrm{mgl}^{-1}$ ART separated or died with a median duration of 7 and 4 days after exposure to ART, respectively. ART significantly reduced the survival times of male (median: 9 days, $P<0.01$ ) and

Table 1. Effect of artesunate on the survival time of Schistosoma mansoni paired adult worms during in vitro incubation.

\begin{tabular}{|c|c|c|c|c|}
\hline \multirow{2}{*}{$\begin{array}{l}\text { Concentration of } \\
\text { artesunate }\left(\mathrm{mg} \mathrm{l}^{-1}\right)\end{array}$} & \multirow{2}{*}{$\begin{array}{l}\text { Number of } \\
\text { worm pairs }\end{array}$} & \multicolumn{2}{|c|}{ Median survival days (range) } & \multirow[b]{2}{*}{$P$ value } \\
\hline & & Male & Female & \\
\hline \multicolumn{5}{|l|}{ Experiment 1} \\
\hline Control & 16 & ND & ND & - \\
\hline 40 & 16 & $3(3-4)^{*}$ & $3(2-3)^{*}$ & $<0.001$ \\
\hline 20 & 16 & $6(5-8)^{*}$ & $4(3-5)^{*}$ & $<0.001$ \\
\hline \multicolumn{5}{|l|}{ Experiment 2} \\
\hline Control & 9 & ND & ND & - \\
\hline 10 & 9 & $9(9-10)^{*}$ & $6(5-7)^{*}$ & $<0.001$ \\
\hline 5 & 9 & $18(12-21)^{*}$ & $9(8-16)^{*}$ & $<0.001$ \\
\hline
\end{tabular}

Statistical analysis used a $\log$ rank test. ${ }^{*} P<0.01$, compared with the corresponding control group. ND, No death was observed in any worms during 8 days and 21 days in Experiment 1 and Experiment 2, respectively. 
female (median: 6 days, $P<0.01$ ) worms at a concentration of $10 \mathrm{mgl}^{-1}$, and also at a concentration of $5 \mathrm{mgl}^{-1}$ (male median: 18 days, $P<0.01$, female median: 9 days, $P<0.01)$ compared with the control group.

Furthermore, as shown in table 1, ART significantly reduced the survival times of female worms at concentrations of 5,10, 20 and $40 \mathrm{mgl}^{-1}$ compared with the survival times of male worms.

\section{The effect of artesunate on egg production}

The effect of ART on the daily egg output by paired female adult worms is shown in fig. 1. In Experiment 1 (fig. 1a), the mean daily egg output during 1 day preincubation in the control group $(n=16)$ was $13.6 \pm 2.7$. On the second day of incubation, the mean daily egg output reached a peak of $80.6 \pm 7.0$, and decreased daily thereafter. The mean daily egg output during 1 day preincubation was $14.0 \pm 2.8$ and $14.9 \pm 3.5$ in the 20 and $40 \mathrm{mg} \mathrm{l}^{-1}$ ART groups, respectively. On the first day after
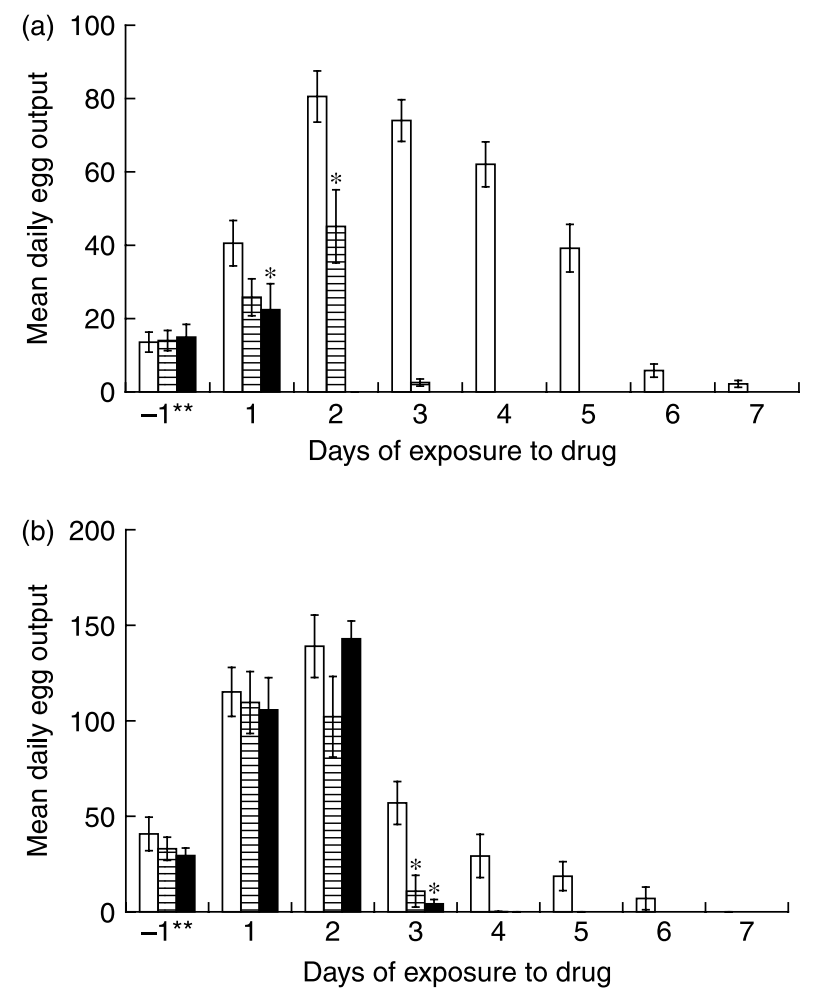

Fig. 1. Effect of artesunate on daily egg output of paired adult Schistosoma mansoni worms in vitro. (a) Sixteen adult worm pairs were incubated with artesunate at concentrations of $0 \mathrm{mgl}^{-1}$

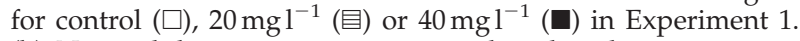
(b) Nine adult worm pairs were incubated with artesunate at concentrations of $0 \mathrm{mgl}^{-1}$ for control $(\square), 5 \mathrm{mgl}^{-1}$ (目) or $10 \mathrm{mgl}^{-1}(\mathbf{\square})$ in Experiment 2. Each bar shows the arithmetic mean \pm standard error of daily egg output. ${ }^{*} P<0.05$, compared with the corresponding control group by MannWhitney test. ${ }^{* *} 1$ day incubation in medium alone before worms were exposed to the drug. exposure to ART, the mean daily egg output was $40.6 \pm 6.2$ in the control group, and $25.8 \pm 5.0$ and $22.4 \pm 7.1$ in the 20 and $40 \mathrm{mg}^{-1}$ ART groups. The daily egg output was significantly lower in the $40 \mathrm{mg} \mathrm{l}^{-1}$ ART group than in the control group $(P<0.01)$. On the second day, egg production was zero in this group. The mean daily egg output in the $20 \mathrm{mgl}^{-1}$ ART group on the second day was $45.1 \pm 10.0$ and was also significantly lower than that of the control group $(P<0.01)$. Egg production was completely halted in this group by day 4 .

In Experiment 2 (fig. 1b), the mean daily egg output during 1 day pre-incubation in the control group $(n=9)$ was $40.8 \pm 8.8$. On the second day of incubation, the mean egg output reached a peak of $139.0 \pm 16.3$, before decreasing daily. The mean daily egg output during 1 day pre-incubation was $33.0 \pm 6.1$ and $29.4 \pm 4.0$ in the 5 and $10 \mathrm{mgl}^{-1}$ ART groups, respectively. The mean daily egg output on the first day after exposure to ART was $115.1 \pm 12.9$ in the control group, $109.6 \pm 16.2$ and $105.7 \pm 16.9$ in the 5 and $10 \mathrm{mgl}^{-1}$ ART groups. On the second day of drug administration these values were $139.0 \pm 16.3$ for the control group, and $102.1 \pm 21.1$ and $149.7 \pm 13.4$ in the 5 and $10 \mathrm{mgl}^{-1}$ ART groups, respectively. During the first and second days post exposure to ART, there was no significant difference in daily egg output between both ART groups and the control group. On the third day, the mean daily egg output in the 5 and $10 \mathrm{mgl}^{-1}$ ART groups was $10.8 \pm 8.3$ and $4.8 \pm 2.1$, respectively. Daily egg output was significantly lower in the $5 \mathrm{mgl}^{-1}(P<0.001)$ and $10 \mathrm{mgl}^{-1}(P<0.001)$ ART groups than in the control group. On the fourth day, egg output continued in the control group, while both ART groups had completely stopped producing eggs.

\section{Apparent alterations in morphology of adult Schistosoma mansoni worms caused by artesunate}

The appearance of adult worms exposed to ART was observed daily under a dissecting microscope. When adult worms were exposed to 20 or $40 \mathrm{mgl}^{-1}$ ART, small vesicles appeared focally on the tegumental surface of male worms as early as 4 or 2 days, respectively. These vesicles grew larger over time, and at the end of cultivation the adult worms showed a rough tegumental surface. Similar tegumental changes were also observed in male worms exposed to the lower concentrations of ART.

\section{Discussion}

When worm pairs of $S$. mansoni were exposed to ART in monotherapy at low concentrations of 20 or $40 \mathrm{mgl}^{-1}$, all worms died within 8 or 4 days, respectively. ART also significantly reduced the survival times of male and female worms at lower concentrations of 5 or $10 \mathrm{mgl}^{-1}$. Compared to the results of Xiao \& Catto (1989), and Xiao et al. (2001), the effect of ART on adult worms was observed in vitro at lower concentrations.

On the other hand, Utzinger et al. (2002) showed that ARM has higher antischistosomal activities than ART in a mouse model, and explained these differences in 
antischistosomal activity in relation to differences in the rate of metabolism of the two drugs. ART is absorbed rapidly and metabolized to dihydroartemisinin (DHA) at a high rate, whereas, following intramuscular injection, oil-soluble ARM is slowly and erratically absorbed, with relatively little conversion to DHA (Hien et al., 2004).

In contrast, drugs are not metabolized in vitro as they are in vivo. ART is a water-soluble sodium hemisuccinyl ester, whereas ARM is an oil-soluble methyl ether of DHA. The different properties of these two drugs might directly influence their effects on adult S. mansoni worms in vitro. Incubation conditions such as medium and its exchanging period in the present study were different from those in the study of Xiao \& Catto (1989), and Xiao et al. (2001), and so the two studies cannot be directly compared.

We found that female worms were more sensitive to ART than males, a finding consistent with a previous study by Lescano et al. (2004), who found that the recovery rate of female worms was notably reduced in mice treated with ARM (50 or $100 \mathrm{mg} \mathrm{kg}^{-1}$ ) on the twentieth day after infection.

Xiao and Catto (1989) also suggested that degeneration of the reproductive organs of worms caused by ARM may inhibit egg formation. However, there have been no previous reports related to assessing the effect of an ARS derivative on daily egg output in worms cultured in vitro. Here, we found that daily egg output was significantly reduced 1 day after exposure to $40 \mathrm{mgl}^{-1}$ ART, and was completely stopped by day 2 . In vitro exposure of worm pairs to $20 \mathrm{mgl}^{-1}$ ART also significantly decreased the daily egg output within 2 days. Reduction of daily egg output was also observed at concentrations of 5 or $10 \mathrm{mgl}^{-1}$ of ART, after 3 days' exposure in vitro. The direct effects of ART on the daily egg output and survival times of paired adult worms were dependent on concentration and duration of exposure to the drug. ART appeared to affect egg production earlier than it affected survival time.

Xiao \& Catto (1989) showed that ARM retarded the growth of both male and female S. mansoni worms, and also caused the degeneration of the reproductive organs of worms in a mouse model, whereas ARM alone had no apparent action on schistosomes in vitro at concentrations below $100 \mathrm{mgl}^{-1}$. Subsequently, Xiao et al. (2001) demonstrated that almost all worms died after $48-72 \mathrm{~h}$ in vitro incubation with 5 or $10 \mathrm{mgl}^{-1} \mathrm{ARM}$ and 50 or $100 \mathrm{mgl}^{-1}$ haemin. Worms exposed to ARM combined with haemin showed a gradual decrease in motor activities. Small vesicles appeared focally on the tegumental surface of worms, grew larger, and eventually collapsed. These alterations in appearance of the surface of worms, caused by a combination of ARM with haemin, were similar to the alterations observed in the present experiment with ART monotherapy. Xiao et al. (2001) suggested that ARM is activated within schistosomes by haem or other iron compounds as it is in malaria parasites, producing toxic compounds and/or free radicals, as suggested by Butler et al. (1998). A similar mode of action may also account for the antischistosomal activities of ART. Thus, it is reasonable to suggest that ART is activated by haem within worms to produce free radicals and electrophilic intermediates.
These reactive species might be responsible for damaging reproductive organs or killing worms.

Since ARS derivatives are promising antischistosomiasis drugs, clinical trials have already evaluated and proved their prophylactic protection against schistosomiasis (Utzinger et al., 2000; Xiao et al., 2000; Jiraungkoorskul et al., 2006). Currently, field trials are being carried out to evaluate the efficacy and safety of combination therapy of PZQ and ART against S. mansoni in Senegal and S. haematobium in Gabon (Borrmann et al., 2001; De Clercq et al., 2006). Considering this, our present findings may contribute to the current strategic discussions on schistosomiasis treatment and control. However, great care must be taken when administering ARS derivatives in areas where malaria is endemic (Utzinger et al., 2000), as this may increase the chances of selecting resistance in malaria parasites.

\section{Acknowledgements}

We wish to thank Dr Masahiro Hashizume, Department of International Health, Institute of Tropical Medicine, Nagasaki University for his statistical advice and Dr Richard Culleton, Department of Protozoology, Institute of Tropical Medicine, Nagasaki University for useful suggestions and comments on the manuscript.

\section{References}

Borrmann, S., Szlezak, N., Faucher, J.F., Matsiegui, P.B., Neubauer, R., Binder, R.K., Lell, B. \& Kremsner, P.G. (2001) Artesunate and praziquantel for the treatment of Schistosoma haematobium infections: a double-blind, randomized, placebo-controlled study. Journal of Infectious Diseases 184, 1363-1366.

Butler, A.R., Gilbert, B.C., Hulme, P., Irvine, L.R., Renton, L. \& Whitwood, A.C. (1998) EPR evidence for the involvement of free radicals in the iron-catalysed decomposition of qinghaosu (artemisinin) and some derivatives; antimalarial action of some polycyclic endoperoxides. Free Radical Research 28, 471-476.

Chitsulo, L., Engels, D., Montresor, A. \& Savioli, L. (2000) The global status of schistosomiasis and its control. Acta Tropica 77, 41-51.

De Clercq, D., Vercruysse, J., Verle, P., Kongs, A. \& Diop, M. (2000) What is the effect of combining artesunate and praziquantel in the treatment of Schistosoma mansoni infections? Tropical Medicine and International Health 5, 744-746.

De Clercq, D., Vercruysse, J., Kongs, A., Verlé, P., Dompnier, J.P. \& Faye, P.C. (2002) Efficacy of artesunate and praziquantel in Schistosoma haematobium infected schoolchildren. Acta Tropica 82, 61-66.

Doenhoff, M.J., Kusel, J.R., Coles, G.C. \& Cioli, D. (2002) Resistance of S. mansoni to praziquantel: is there a problem? Transactions of the Royal Society of Tropical Medicine and Hygiene 96, 465-469.

Guisse, F., Polman, K., Stelma, F.F., Mbaye, A., Talla, I., Niang, M., Deelder, A.M., Ndir, O. \& Gryseels, B. (1997) Therapeutic evaluation of two different dose regimens of praziquantel in a recent Schistosoma 
mansoni focus in Northern Senegal. American Journal of Tropical Medicine and Hygiene 56, 511-514.

Hien, T.T., Davis, T.M., Chuong, L.V., Ilett, K.F., Sinh, D.X., Phu, N.H., Agus, C., Chiswell, G.M., White, N.J. \& Farrar, J. (2004) Comparative pharmacokinetics of intramuscular artesunate and artemether in patients with severe falciparum malaria. Antimicrobial Agents and Chemotherapy 48, 4234-4239.

Ismail, M., Botros, S., Metwally, A., William, S., Farghally, A., Tao, L.F., Day, T.A. \& Bennett, J.L. (1999) Resistance to praziquantel: direct evidence from Schistosoma mansoni isolated from Egyptian villagers. American Journal of Tropical Medicine and Hygiene 60, 932-935.

Jiraungkoorskul, W., Sahaphong, S., Sobhon, P., Riengrojpitak, S. \& Kangwanrangsan, N. (2006) Schistosoma mekongi: the in vitro effect of praziquantel and artesunate on the adult fluke. Experimental Parasitology 113, 16-23.

Lescano, S.Z., Chieffi, P.P., Canhassi, R.R., Boulos, M. \& Amato Neto, V. (2004) Antischistosomal activity of artemether in experimental Schistosomiasis mansoni. Revista de Saúde Pública 38, 71-75.

Magnussen, P. (2003) Treatment and re-treatment strategies for schistosomiasis control in different epidemiological settings: a review of 10 years' experiences. Acta Tropica 86, 243-254.

Montresor, A., Crompton, D.W.T., Gyorkos, T.W. \& Savioli, L. (2002) Helminth control in school-age children: a guide for managers of control programmes. Geneva, World Health Organization.

Smithers, S.R. \& Terry, R.J. (1965) The infection of laboratory hosts with cercariae of Schistosoma mansoni and the recovery of the adult worms. Parasitology 55, 695-700.
Utzinger, J., N'Goran, E.K., N'Dri, A., Lengeler, C., Xiao, S. \& Tanner, M. (2000) Oral artemether for prevention of Schistosoma mansoni infection: randomised controlled trial. Lancet 355, 1320-1325.

Utzinger, J., Chollet, J., You, J., Mei, J., Tanner, M. \& Xiao, S. (2001) Effect of combined treatment with praziquantel and artemether on Schistosoma japonicum and Schistosoma mansoni in experimentally infected animals. Acta Tropica 80, 9-18.

Utzinger, J., Chollet, J., Tu, Z., Xiao, S. \& Tanner, M. (2002) Comparative study of the effects of artemether and artesunate on juvenile and adult Schistosoma mansoni in experimentally infected mice. Transactions of the Royal Society of Tropical Medicine and Hygiene 96, 318-323.

Xiao, S.H. \& Catto, B.A. (1989) In vitro and in vivo studies of the effect of artemether on Schistosoma mansoni. Antimicrobial Agents and Chemotherapy 33, 1557-1562.

Xiao, S.H., You, J.Q., Yang, Y.Q. \& Wang, C.Z. (1995) Experimental studies on early treatment of schistosomal infection with artemether. The Southeast Asian Journal of Tropical Medicine and Public Health 26, 306-318.

Xiao, S.H., Booth, M. \& Tanner, M. (2000) The prophylactic effects of artemether against Schistosoma japonicum infections. Parasitology Today 16, 122-126.

Xiao, S., Chollet, J., Utzinger, J., Matile, H., Mei, J. \& Tanner, M. (2001) Artemether administered together with haemin damages schistosomes in vitro. Transactions of the Royal Society of Tropical Medicine and Hygiene 95, 67-71.

(Accepted 30 July 2009)

First published online 10 October 2008 (C) 2009 Cambridge University Press 